\title{
Effect of Periodontal Therapy on Alveolar Bone as Measured by Subtraction Radiography*
}

\author{
Edgar F. Schmidt, † Richard L. Webber, $\ddagger$ Urs E. Ruttimann $\ddagger$ and \\ Walter J. Loesche†
}

Accepted for publication 30 October 1987

\begin{abstract}
ChANGES IN THE PERIOdONTAL ALVeOlar bone are often evaluated by comparing a series of radiographs taken over time. This investigation used a technique that allowed the image registration to be geometrically standardized each time a radiograph was taken. Radiographs of 24 patients from an ongoing double-blind, clinical study using metronidazole were obtained: (1) before any treatment, (2) at the completion of scaling and root planing and surgery (when performed) and (3) during the maintenance phase. One hundred six (106) paired comparisons were analyzed by subtraction radiography using a computerized system. Of these, $95(89 \%)$ exhibited a minimal degree of geometric distortion and could be successfully substracted. Most areas (67\%) showed no change in bone structure following periodontal treatment. Bone gain was noted in $12 \%$ of the sites examined, while bone loss was seen in $21 \%$ of the sites. This bone loss was statistically associated with sites that had received some form of surgical treatment.
\end{abstract}

Radiographs are the only noninvasive method of detecting changes in the periodontal alveolar bone. In recent years procedures have been described, which permit the computer comparison of standardized radiographs taken over time. In order to compare by a subtraction technique, the images must be registered with a minimum degree of geometric distortion. Several attempts have been made at standardizing the geometric projections of radiographs. ${ }^{1-6}$ One method $^{7}$ that described a beam-guiding device connected to the teeth and film, but uncoupled from the $\mathrm{x}$-ray source, employed an occlusal registration to position the film in addition to means for assessing angulation differences in image registration. In the present investigation, this method was modified by introducing a connecting device that would mechanically couple the $\mathrm{x}$-ray source, film and teeth in a safe and practical way to produce multiple, geometrically standardized $\mathrm{x}$-ray registrations. Radiographs were obtained by this modified technique in patients participating in a double-blind, clinical trial of metronidazole. These radiographs were analyzed and

\footnotetext{
* This research was supported by Public Health grants DE02731 and DE06030 from the National Institute of Dental Research.

$\dagger$ Department of Oral Biology, The University of Michigan, School of Dentistry, Ann Arbor, MI 48109. Present address: University of Detroit, Department of Oral Medicine/Periodontics, Detroit, MI 48207.

$\ddagger$ Diagnostic Systems Branch, National Institute of Dental Research, National Institutes of Health, Bethesda, MD 20205.
}

compared by a computerized subtraction technique in order to observe any effects of periodontal therapy with and without the use of systemic metronidazole and with and without periodontal surgery on the alveolar bone.

\section{MATERIALS AND METHODS}

Customized bite blocks were constructed as described by Duckworth et al. ${ }^{7}$ Briefly, occlusal registrations of the area of interest were obtained by having the patient bite on a polyether impression material§ that was placed on a vertical bite-wing film holder. $\|$ Density standardization was provided by a copper stepwedge that was attached to the end of the film holder by heating the wedge to melt into the plastic. One square and one round orthodontic wire were placed on the mandibular surface of the film holder at a known distance from the film (square $10 \mathrm{~mm}$, round $30 \mathrm{~mm}$ ) to determine the angular error. On the maxillary surface of the film holder, an orthodontic wire (0.30-foot diameter) was placed parallel to the central groove of the teeth to provide a calibration standard for image magnification. A metal sheath was inserted on the back of the film to increase rigidity and to improve the image contrast.

The angular error was controlled by observing the registration of the round and square orthodontic wires

§ Polygel, L. D. Caulk Co, Milford, DE 19963.

॥ VIP System; The Uprod Corp, Ft. Lauderdale, FL 33307. 
on the film. These wires were placed coaxially on the perpendicular, so that a perfect superimposition of the round and square dots on the film would indicate that both vertical and horizontal angulations of the cone were perpendicular with the film (the placement of a metal sheath to increase rigidity of the film was important in this aspect). If the round and square dots on the film were found to be more than $2 \mathrm{~mm}$ apart, (either vertically, horizontally or both), the radiograph was retaken. This was assessed during our calibration stage when radiographs with such angular error could not be successfully subtracted. Such an occurrence was rare when the patient's occlusion was intact. However, when crowns or fixed-prostheses were placed after the initial bite registrations had been taken, the bite block was at times unstable and positioning inaccurate.

The film holder was connected to the $x$-ray cone by means of an O-ring seal created by suction. A bracket fixed around the $x$-ray cone contained a groove that embraced a rubber O-ring. The O-ring was put in contact with the flat outer surface of a plastic connector, which was attached at its other end to the film holder (Fig. 1). The system was aligned and then fixed in place by producing a vacuum in the O-ring groove by aspirating air through a portal on the bracket, which was connected by tubing to the high power suction mechanism of the dental unit. Vacuum grease was applied to both contacting surfaces to ensure an adequate seal. The coupling of the $x$-ray film, teeth and $x$-ray cone in a fixed relationship (Fig. 2) allowed image registration to be geometrically standardized each time a radiograph was taken. If the seal was broken by any unexpected movement of the patient (such as sneezing or coughing), the coupling of the cone and the connecting device would easily separate thereby preventing any serious injury to the patient's teeth, joints or jaw.

The ability of this O-ring connecting device to pro-

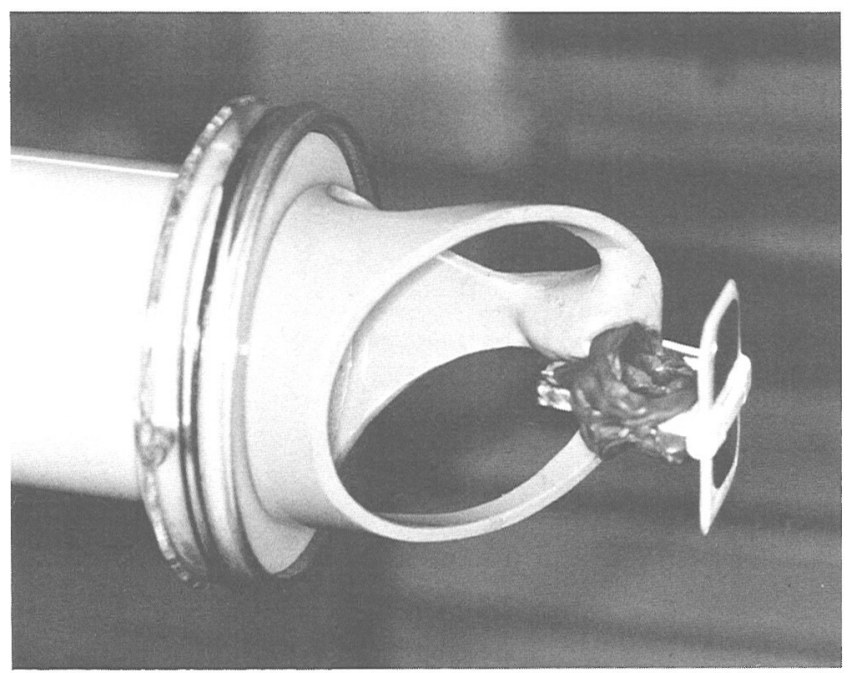

Figure 1. Positioning system used to obtain standardized radiographs. Note that the film holder is connected to $x$-ray cone by an $O$ ring.

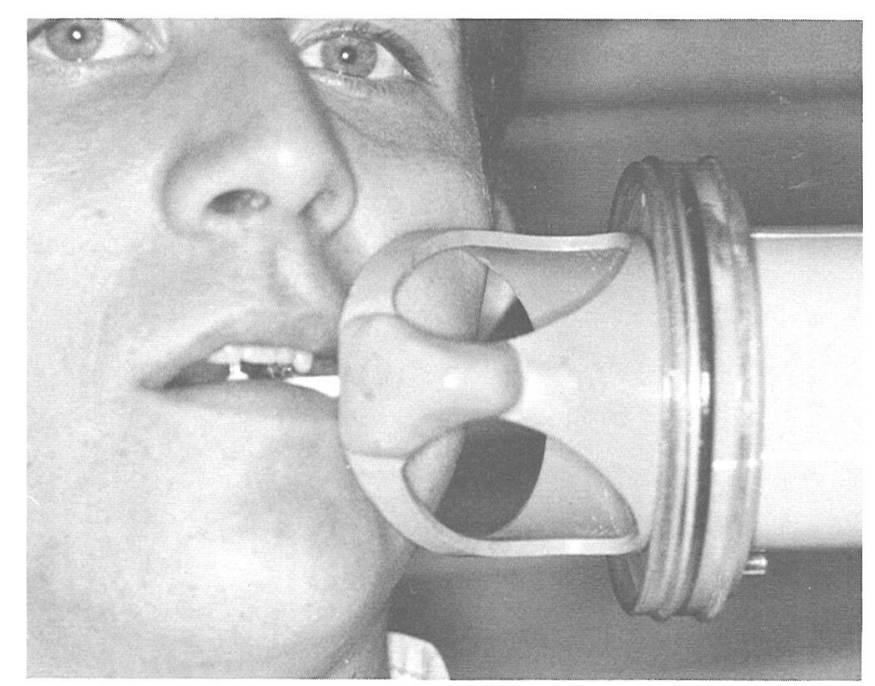

Figure 2. The positioning system in place in the patient.

vide standardized radiographs was evaluated during the course of an ongoing double-blind, clinical trial of the efficacy of systemic metronidazole in the treatment of periodontitis. Patients who had both evidence of an anaerobic subgingival flora dominated by spirochetes and were recommended as needing periodontal surgery were randomly assigned to a metronidazole or a placebo group. The patients received either metronidazole or placebo medication $(250 \mathrm{mg})$ at the first visit in which the root surfaces were debrided and were instructed to take the medication three times a day for one week. The actual taking of the medication was unsupervised. The patients then went on to receive all the necessary root surface debridement and surgery, as required.

Radiographs were obtained at baseline before any treatment was performed, at three to 14 months after completion of periodontal therapy (in which treatment may or may not have included surgery) and again approximately one year later. From two to eight pairs of radiographs were taken per patient depending on the severity of the case and the number of teeth present. Only those radiographs were analyzed that contained evidence of enough trabecular bone in the regions of diagnostic interest to ensure unequivocal bases for registration and whose angulation error was less than $2 \%$ as determined by the apparent separation of the round and square dots produced by the orthodontic wires in the film holder.

The three examiners performing the subtraction analysis (EFS, RLW and UER) had to agree as to which pairs of radiographs were suitable for analysis. In each pair of radiographs, from one to three distinct interproximal areas for a total of 124 were analyzed by subtraction radiography and scored for bone loss or bone gain. Although the criteria used for making this decision depended in part on experience using the described methods, they can be specified in ways that alter the distribution of errors by type (omission vs. commission) as demonstrated by in vitro calibration 
studies. ${ }^{8}$ In this case, each examiner was instructed to superimpose a radiographic image to be subtracted with its negative counterpart exposed during an earlier examination in two separate and distinct steps using a video-based image transduction system.

The first step was to manipulate the paired images such that the resulting composite image of trabecular bone in the region of diagnostic interest seen in real time on a television screen cancelled maximally. This process involved iterative manipulations consisting of subtle rotations and translations of one image relative to the other using a micromanipulator attached to the video system used to digitize the images for subsequent computerized image analysis. If examination of regions in the resulting digital subtractions having a range of radiopacities known to be unchanged from one radiographic exposure to the next (such as the areas containing the copper stepwedge) indicated systematic changes in gray level, then the entire process was repeated after contrast correction using the method described by Ruttimann et al. ${ }^{9}$ Only after this process was successfully completed was the examiner instructed to look for areas of potential bone loss or gain.

The second step involved attempts to alter the registration slightly so as to eliminate, as much as possible, any evidence of bone loss or gain observed in step one while still maintaining a comparable degree of trabecular cancellation in the composite image. Only changes occupying an estimated area of $9 \mathrm{~mm}^{2}$ or larger, that persisted after diligent efforts to get them to cancel while maintaining trabecular registration, were reported as gains or losses in this investigation. Hence, the net effect of these registration criteria was to bias the selection threshold for reported changes in bone toward high specificity at the expense of detection sensitivity.

The pairs of radiographs which showed either bone gain or bone loss in specific sites were compared as a function of either the treatment group the subject was in, i.e., metronidazole or placebo, or as a function of the treatment that the specific site received, i.e., surgery with or without metronidazole; no surgery with or without metronidazole, by a series of two-way comparisons using the Fisher's exact test.

\section{RESULTS}

One hundred six pairs of vertical bitewing radiographs were obtained from 24 patients prior to treatment and at three to 14 months after completion of treatment. Ninety-five pairs $(89 \%)$ of these radiographs could be successfully subtracted and analyzed. Eleven pairs $(10 \%)$ presented either a poor geometric standardization ( 7 pairs) or lacked contrast due to overdevelopment (4 pairs) and could not be subtracted (Table 1). Of the subtracted interproximal areas, 124 could be scored for bone levels. Of these, $50 \%$ exhibited no discernible change in bone levels before and after treatment (Table 2). Seventeen percent of the areas showed
Table 1

Frequency Distribution of the Paired Comparisons and Scores

\begin{tabular}{ccc}
\hline Patient No. & $\begin{array}{c}\text { No. of Paired } \\
\text { Comparisons }\end{array}$ & No. of Scores* \\
\hline 1 & 6 & 10 \\
2 & 3 & 4 \\
3 & 2 & 4 \\
4 & 2 & 2 \\
5 & 3 & 3 \\
6 & 2 & 4 \\
7 & $8(1) \dagger$ & 8 \\
8 & $3(1)$ & 3 \\
9 & 8 & 11 \\
10 & $3(1)$ & 3 \\
11 & $8(1)$ & 8 \\
12 & $6(2)[1] \ddagger$ & 6 \\
13 & 4 & 4 \\
14 & 4 & 4 \\
15 & 2 & 2 \\
16 & $3[3]$ & 3 \\
17 & 4 & 4 \\
18 & 4 & 6 \\
19 & $6(1)$ & 6 \\
20 & $6(1)$ & 5 \\
21 & $4(1)$ & 6 \\
22 & 5 & 7 \\
23 & 5 & 5 \\
24 & 5 & 124 \\
TOTAL & $106(7)[4]$ & \\
\hline
\end{tabular}

* Interproximal areas.

$\uparrow$ Number of pairs in parenthesis, which could not be subtracted due to geometric distortion.

$\$$ Number of pairs in brackets, which could not be subtracted due to lack of contrast (over- or under-developed).

Table 2

Changes in Bone Level 3-14 Months after Periodontal Therapy

\begin{tabular}{ccccccc}
\hline \multicolumn{4}{c}{ Total number of scores } \\
$124(100 \%)$
\end{tabular}

* Areas under study showed no net change as there were equal amounts of bone gain and loss in discrete foci.

discrete foci of both bone loss and bone gain occurring simultaneously in different parts of the interproximal areas, but overall these areas were considered as exhibiting no net change in bone density (shown as scores exhibiting activity in Table 2).

Twenty-one percent of the analyzed interproximal areas exhibited net bone loss that was usually less than $9 \mathrm{~mm}^{2}$, but seven areas had losses greater than $9 \mathrm{~mm}^{2}$ (Table 2). Figure $3 \mathrm{~A}$ is an example of standardized radiographs that, when subtracted, exhibited sites in which bone loss was both greater than $9 \mathrm{~mm}^{2}$ and less than $9 \mathrm{~mm}^{2}$ (Figure 3B). Twelve percent of the sites exhibited a net bone gain that in most areas was less 

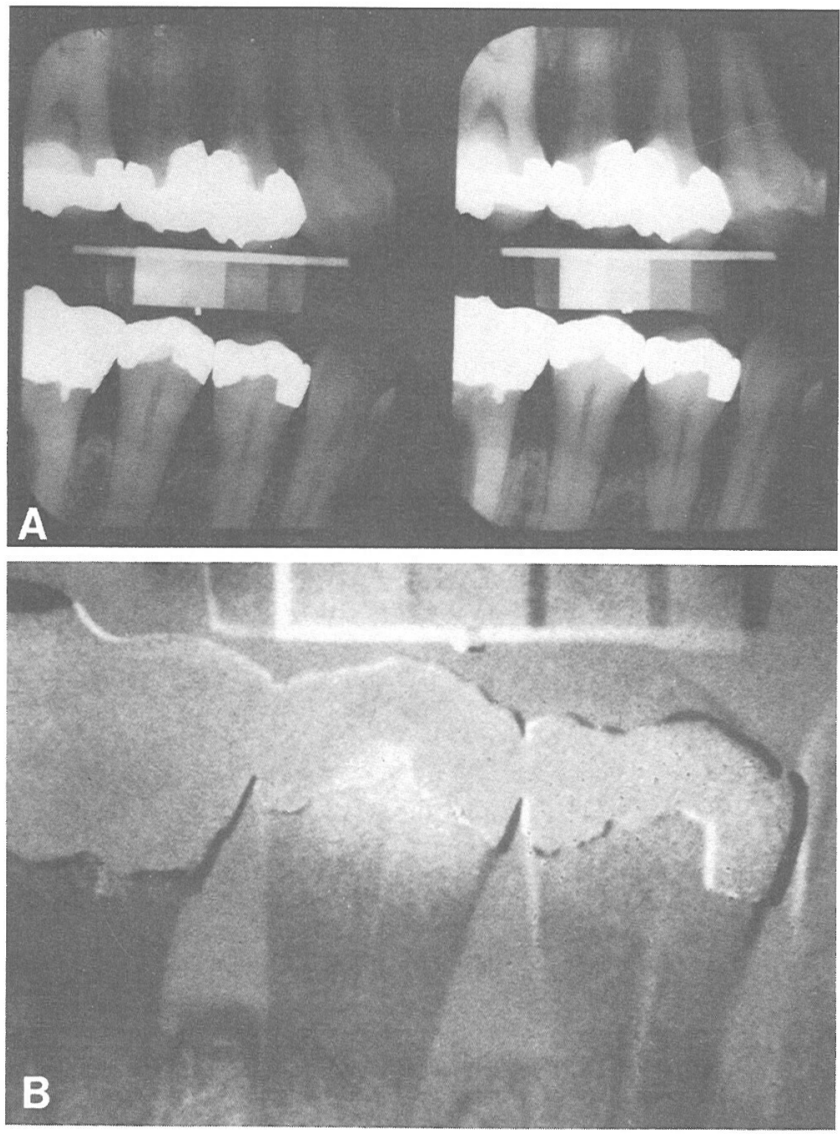

Figure 3. A. A representative pair of standardized radiographs that could be subtracted. The film on the left is a pretreatment radiograph, and the one on the right was taken 15 months later after the patient had completed all phases of treatment, including surgery. B. The substracted image of the radiographs shown in $3 A$ focusing on the lower right quadrant. Note alveolar crestal bone loss between the second premolar and the first molar appearing as a black area. This was a site in which more than $9 \mathrm{~mm}^{2}$ bone loss was observed.

than $9 \mathrm{~mm}^{2}$, but there were four areas in which more than $9 \mathrm{~mm}^{2}$ of bone was gained (Table 2).

The bone scores that exhibited change were analyzed as a function of the type of treatment that the interproximal area had received. All teeth had been scaled and root planed and in addition some of the sites had been surgically treated using a modified Widman flap procedure. This meant that any given interproximal area could have received one of four treatment regimens depending on whether the patient had metronidazole or placebo and whether the interproximal area under investigation had been subjected to surgery or not.

When the areas were compared as to whether they were in the metronidazole-treated patients or the placebo-treated patients, there was no discernible difference between the two treatment regimens (Table 3 ). However, when the areas were compared as to whether they had received surgery or not, bone loss was found in 17 of 18 sites (94\%) that had received flap surgery, and in nine of 23 sites (39\%) that did not undergo surgery (Table 4). This difference between sites that did or did not undergo surgery was statistically significant $(P=0.002$, Fisher's exact test).

The bone scores were then compared as to the interactive effects of surgery and metronidazole (Table 5). Seven of the nine areas that had been exposed to metronidazole treatment without surgery exhibited bone gain, whereas only one of 11 areas that had been exposed to metronidazole plus surgery exhibited bone gain. When a similar comparison was made in the placebo group (positive control) seven of 14 areas that had received scaling and root planing showed bone gain, but none of the seven areas that had received surgical treatment showed any bone gain (Table 6). In both patient groups, the differences between sites that had or had not received surgical treatment were significant $(P<0.05$, Fisher exact test).

Many of the radiographs that were taken approximately one year later during the recall maintenance period could not be successfully subtracted with either the pre-treatment radiographs or with the immediate post-treatment radiographs. This was due to either deterioration of the impressions, tooth loss via extraction, tooth movement or the placement of dental restorations. Thus, it was not possible using these procedures to obtain long term follow-up data of three years or more on bone levels in these interproximal areas.

\section{DISCUSSION}

This investigation used a radiograph positioning technique that allowed the image registration to be geometrically standardized each time a radiograph was taken. The results showed that $89 \%$ of the paired comparisons could be successfully subtracted and analyzed by a computer digitizing system. This high percentage of success relative to other reports in which approximately $60 \%$ of the pretreatment and posttreatment radiographs could be subtracted ${ }^{10}$ was probably due to variations in

Table 3

Effect of Debridement Plus Metronidazole or Debridement Plus Placebo on Changes in Bone Levels

\begin{tabular}{cccc}
\hline Bone changes & Metronidazole* & Positive control $\dagger$ \\
\hline Bone gain & 8 score & 7 score & 15 \\
Bone loss & $\frac{12}{20}$ & $\frac{14}{21}$ & $\frac{26}{41}$ \\
& $P=0.45$ Fisher's exact test & \\
\hline
\end{tabular}

$*$ Metronidazole $=$ metronidazole + scaling and root planing.

$\dagger$ Positive control $=$ placebo + scaling and root planing.

Table 4

Effect of Surgery on Changes in Bone Levels

\begin{tabular}{cccc}
\hline \multirow{2}{*}{ Bone changes } & \multicolumn{3}{c}{ Scores } \\
\cline { 2 - 4 } & Surgery & No surgery & Tötals \\
\hline Bone gain & 1 & 14 & 15 \\
Bone loss & $\frac{17}{18}$ & $\frac{9}{23}$ & $\frac{26}{41}$ \\
& Fisher's exact test $P=0.0002$ & \\
\hline
\end{tabular}


Table 5

Effect of Surgery and/or Metronidazole on Sites that Showed Changes in Bone Levels

\begin{tabular}{cccc}
\hline \multirow{2}{*}{ Bone changes } & \multicolumn{3}{c}{ Scores } \\
\cline { 2 - 4 } & Metro* $^{\text {Metro* }+ \text { Surgery } \dagger}$ & Totals \\
\hline Bone gain & 7 & 1 & 8 \\
Bone loss & $\frac{2}{9}$ & $\frac{10}{11}$ & $\frac{12}{20}$ \\
& Fisher exact test $P=0.003$ & \\
\hline
\end{tabular}

* Metro $=$ metronidazole + scaling and root planing.

$\dagger$ Surgery $=$ modified Widman flap.

Table 6

Effect of Surgery on Sites that Showed Changes in Bone Level

\begin{tabular}{cccc}
\hline \multirow{2}{*}{ Bone changes } & \multicolumn{3}{c}{ Scores } \\
\cline { 2 - 4 } & Positive control* & $\begin{array}{c}\text { Positive control* } \\
+ \text { surgery } \dagger\end{array}$ & Totals \\
\hline Bone gain & 7 & 0 & 7 \\
Bone loss & $\frac{7}{14}$ & $\frac{7}{7}$ & $\frac{14}{21}$ \\
& Fisher exact test $P=0.03$ & \\
\hline
\end{tabular}

* Positive control $=$ placebo + scaling and root planing.

$\uparrow$ Surgery $=$ modified Widman flap.

acceptance criteria, although the vacuum seal method for reproducibly coupling the film-teeth-cone complex may have played a significant role by keeping angulation errors to a minimum. While this vacuum seal was rigid enough to ensure stability and accuracy, it would separate easily with any unexpected movement of the patient, such as coughing or sneezing. No patient complaints of discomfort or uneasiness were heard during the entire course of the study.

The impression material began to peel off some of the bite blocks over time. This was not a problem when radiographs were obtained within 18 to 24 months after the bite block had been fabricated, but was a problem in some radiographs taken 30 to 36 months after the construction of the bite block. This combined with possible tooth movement, placement of restorations and tooth loss resulted in the inability to subtract many of the radiographs taken in the year subsequent to the data reported in this investigation. This suggests that alternative techniques not requiring occlusal impressions to reproduce radiograph positions need to be developed for studies that will last three or more years.

Current data suggest that the destructive path of periodontal disease may be episodic rather than linear and that the majority of sites observed over a period of one to three years showed no change in attachment levels. ${ }^{11-14}$ This is in agreement with the present results in which $67 \%$ of the areas that could be examined showed no difference in the bone level or density over the one- to two-year period of observation. Of the 26 areas that demonstrated bone loss, $17(65 \%)$ were sites that had been treated with Widman flap surgery, whereas of the 15 areas that showed bone gain, only one $(7 \%)$ was from a site that had been treated surgically (Table 4).

This indicates that surgery involving a Widman flap procedure was more likely to be associated with bone loss than bone gain in these patients. Other studies have shown that bone loss at the alveolar crest following fullthickness flap surgery in humans ranged from 0.5 to 1 $\mathrm{mm}$ and that it varied with flap adaptation, surgical trauma and thickness of both the bone and the flap. The resorbed bone has a tendency to regenerate so as to approach preoperative bone levels within ten weeks. ${ }^{15-20}$ All of our postsurgical radiographs were taken at least 12 weeks after the last surgery so that our observations should be beyond the time period that any remodeling was occurring and thus would reflect the final healing response following surgery.

Fourteen sites that did not receive surgical treatment had evidence of bone gain (Table 4). Seven sites occurred in the metronidazole plus debridement patients (Table 5), and seven occurred in the placebo plus debridement patients (Table 6), which would suggest no difference between these treatments. However, in these nonsurgerized sites, two of nine (22\%) showed bone loss in the metronidazole patients (Table 5), compared to seven of $14(50 \%)$ in the placebo patients (Table 6). While the difference between groups was not significant, this tendency for bone gain with metronidazole had been suggested previously on nonstandardized radiographs. ${ }^{21}$

In conclusion, the radiograph positioning technique described in this study provided standardized radiographs that exhibited a high degree of geometric standardization as witnessed by their suitability for computerized subtraction analysis. However, this reproducibility was lost after periods of two years or more due to deterioration of the impression material and tooth movement. Short-term longitudinal studies evaluating the therapy, activity and epidemiology of dental diseases may benefit from this positioning technique. Studies of a longer duration may require other positioning devices, such as a cephalostat (M. K. Jeffcoat, personal communication). However, even with this procedure it will take several years of clinical evaluation to ascertain as to whether reliable and reproducible standardized radiographs can be obtained over time.

\section{REFERENCES}

1. Dawkins, J.: An apparatus for obtaining serial roentgenograms. Aust Dent J 3: 283, 1958.

2. Benkow, H. H.: Appliance for identical radiography and stereoradiography. Dent Radiogr Photogr 30: 21, 1957.

3. Dalitz, G. D.: A variation of apparatus for obtaining serial roentgenograms. Aust Dent J 9: 29, 1964.

4. Renggli, H. H., Steiner, E., and Curilovic, Z.: Reproducible radiographs and photographs in periodontal diagnosis. Paradontologie 25: $66,1971$.

5. Rosling, B., Hollender, L., Nyman, S., and Olson, G.: A radiographic method for assessing changes in alveolar bone height following periodontal therapy. J Clin Periodontol 2: 211, 1975. 
6. Hausmann, E., McHenry, K., Christenson, L., et al.: Techniques for assessing alveolar bone mass changes in periodontal disease with emphasis on ${ }^{125} \mathrm{I}$ absorptiometry. $J$ Clin Periodontol 10: 455, 1983.

7. Duckworth, J. E., Judy, P. F., Goodson, J. M., and Socransky, S. S.: A method for the geometric and densitometric standardization of intraoral radiographs. $J$ Periodontol 54: 435, 1983.

8. Grondahl, K., Grondahl, H. G., and Webber, R. L.: Influence of variations in projection geometry on the detectability of periodontal bone lesions. J Clin Periodontol 11: 411, 1984.

9. Ruttimann, U. E., Webber, R. L., and Schmidt, E. F.: A robust digital method for film contrast correction in subtraction radiography. $J$ Periodont Res 21: 486, 1986.

10. McHenry, K., Hausmann, E., Wikesjö, U., et al.: Methodological aspects and quantitative adjuncts to computerized subtraction radiography. J Periodont Res 22: 125, 1987.

11. Goodson, J. M., Tanner, A. C. R., Haffajee, A. D., et al.: Patterns of progression and regression of advanced destructive periodontal disease. J Clin Periodontol 9: 472, 1982.

12. Haffajee, A. D., Socransky, S. S., and Goodson, J. M.: Comparison of different data analyses for detecting changes in attachment level. J Clin Periodontol 10: 298, 1983.

13. Lindhe, J., Haffajee, A. D., and Socransky, S. S.: Progression of periodontal disease in adult subjects in the absence of periodontal therapy. J Clin Periodontol 10: 433, 1983.
14. Goodson, J. M.: Clinical measurements of periodontitis. $J$ Clin Periodontol 9: 446, 1986.

15. Donnenfeld, O. W., Marks, R. M., and Glickman, I.: The apically positioned flap-a clinical study. J Periodontol 35: 381, 1984.

16. Friedman, N., and Levine, H.: Experimental periodontal surgery on human beings. A clinical histologic (preliminary) study. $J$ Dent Res 43: 791, 1964

17. Kohler, C. A., and Ramfjord, S. P.: Healing of gingival mucoperiosteal flaps. Oral Surg 13: 89, 1960.

18. Tavitigian, R.: The height of the facial radicular alveolar crest following apically repositioned flap operation. J Periodontol. 41: 412, 1970 .

19. Wood, D. L., Hoag, P. A., Donnenfeld, O. W., et al.: Alveolar crest reduction following full and partial thickness flaps. J Periodontol 43: $141,1972$.

20. Ramfjord, S. P., and Ash, M. M.: Periodontology and Periodontics, ed 1, pp 594. Philadelphia, W. B. Saunders Co., 1979.

21. Loesche, W. J., Syed, S. A., Morrison, E. C., et al.: Metronidazole in periodontitis. I. Clinical and bacteriological results after 15 to 30 weeks. J Periodontol 55: 325, 1984.

Send reprint requests to: Dr. Walter J. Loesche, Department of Oral Biology, The University of Michigan, School of Dentistry, Ann Arbor, MI 48109-1078. 